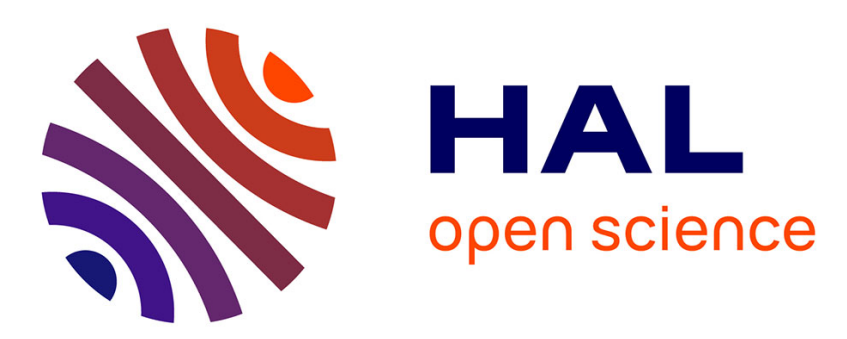

\title{
EFFECT OF PRESSURE AND ELECTRIC FIELD ON CDW INDUCED RESISTIVITY ANOMALIES IN $\mathrm{NbSe} 3$
}

P. Haen, G. Waysand, G. Boch, A. Waintal, P. Monceau, N. Ong, A. Portis

\section{- To cite this version:}

P. Haen, G. Waysand, G. Boch, A. Waintal, P. Monceau, et al.. EFFECT OF PRESSURE AND ELECTRIC FIELD ON CDW INDUCED RESISTIVITY ANOMALIES IN NbSe3. Journal de Physique Colloques, 1976, 37 (C4), pp.C4-179-C4-182. 10.1051/jphyscol:1976430 。 jpa-00216544

\section{HAL Id: jpa-00216544 https://hal.science/jpa-00216544}

Submitted on 1 Jan 1976

HAL is a multi-disciplinary open access archive for the deposit and dissemination of scientific research documents, whether they are published or not. The documents may come from teaching and research institutions in France or abroad, or from public or private research centers.
L'archive ouverte pluridisciplinaire HAL, est destinée au dépôt et à la diffusion de documents scientifiques de niveau recherche, publiés ou non, émanant des établissements d'enseignement et de recherche français ou étrangers, des laboratoires publics ou privés. 


\title{
EFFECT OF PRESSURE AND ELECTRIC FIELD ON CDW INDUCED RESISTIVITY ANOMALIES IN $\mathrm{NbSe}_{3}(*)$
}

\author{
P. HAEN and G. WAYSAND (**) \\ Centre de Recherches sur les Très Basses Températures \\ C. N. R.S., BP 166, 38042 Grenoble-Cédex, France
}

G. BOCH and A. WAINTAL

Laboratoire de Rayons X and Service National des Champs Intenses

B. P. 166 Centre de Tri, 38042 Grenoble-Cédex, France

P. MONCEAU (***), N. P. ONG and A. M. PORTIS

Department of Physics, University of California, Berkeley, CA 94720

\begin{abstract}
Résumé. - Nous présentons une étude sous pression des anomalies qui apparaissent dans les mesures de résistivité à $145 \mathrm{~K}$ et $59 \mathrm{~K}$ dans le composé $\mathrm{NbSe}_{3}$. Ces anomalies sont supposées être dues à l'apparition d'ondes de densité de charge. Les températures de transition décroissent avec la pression avec la même pente $\mathrm{d} T / \mathrm{d} p=4 \mathrm{~K} / \mathrm{kbar}$. Nous avons aussi observé la suppression de ces deux anomalies par l'application d'un champ électrique que nous attribuons à un effet tunnel à travers des gap extrêmement petits introduits par les ondes de densité de charge.
\end{abstract}

\begin{abstract}
We report the effect of pressure on the two resistivity anomalies at $145 \mathrm{~K}$ and $59 \mathrm{~K}$ in $\mathrm{NbSe}_{3}$ and resulting from charge density wave formation. The rate of decrease of the critical temperature with pressure is the same at both transitions ( $\mathrm{d} T / \mathrm{d} p=4 \mathrm{~K} / \mathrm{kbar}$ ). We observe also the suppression by electric fields of these two anomalies, which we attribute to Zener breakdown across extremely small gaps introduced by the CDW.
\end{abstract}

It was very recently reported $[1,2]$ that the electrical resistivity of the transition metal trichalcogenide, $\mathrm{NbSe}_{3}$ showed two phase transitions at $145 \mathrm{~K}$ and $59 \mathrm{~K}$. The onset of charge density waves (CDW) has been suggested to explain these anomalous transport properties [1, 2]. When a CDW forms, gaps open at the Fermi surface (FS) at those portions that satisfy the nesting condition. These gaps reduce the area and change the topology of the FS, leading to an increase in resistivity. Such anomalies in transport properties have been measured in layered dichalcogenides where CDW have been directly observed by electronic diffraction [3]. In $\mathrm{Cr}$ at the Néel temperature the resistivity anomaly has been attributed to spin density waves (SDW) [4]. In this paper we present the results of two experiments which indicate that gaps are induced by $\mathrm{CDW}$ in $\mathrm{NbSe}_{3}$. We report the electrical conductivity measurements under hydrostatic pressure up to $6 \mathrm{kbar}$. The critical temperatures for the formation of the CDW decrease with applied pressure. We report

(*) Supported in part by the National Science Foundation through the Division of Materials Research, Grant 74-23018.

(**) Permanent address : Laboratoire de Physique des Solides, Université Paris-Sud, 91405 Orsay, France.

(***) Permanent address : Centre de Recherches sur les Très Basses Températures, CNRS, B. P. 166, 38042 Grenoble-Cédex, France. also the observation of suppression by electric fields of the anomalies. The nonlinearity of the conductivity with electric field agrees with a Zener-breakdown analysis across extremely small gaps induced by the CDW.

The samples were prepared at Laboratoire de Chimie Minérale, Nantes, France. $\mathrm{NbSe}_{3}$ crystallizes in the form of fibrous strands [5]. Six Se atoms form the vertices of a right triangular prism with a $\mathrm{Nb}$ atom at the center of the prism. Six prisms form a monoclinic unit cell, which measures $10.006 \AA \times 3.478 \AA \times 15.626 \AA$. The distance between $\mathrm{Nb}$ atoms is $3.478 \AA$ along the $b$ axis and varies from $4.45 \AA$ to $4.25 \AA$ in the $a c$ plane $\left({ }^{1}\right)$. The room temperature resistivity has been reported to be $600 \mu \Omega \mathrm{cm}$, and the resistance ratio between $300 \mathrm{~K}$ and $4.2 \mathrm{~K}$ lies between 30 and 40 [1]. Two phase transitions were observed at $145 \mathrm{~K}$ and $59 \mathrm{~K}$, where a sharp increase in resistivity occurs. The resistivity shows maxima for $125 \mathrm{~K}$ and $49 \mathrm{~K}$. The amplitude of the peaks is $10 \%$ of the room temperature resistivity for the higher temperature peak and $30 \%$ for the lower one.

(1) The X-ray data at $4.2 \mathrm{~K}$ are the same as that at room temperature [10]. This indicates that only a slight thermal contraction of the lattice of the order of $0.2 \%$ occurs between room temperature and $4.2 \mathrm{~K}$. 
We have measured the electrical resistivity under hydrostatic pressure. The pressure cell used for this experiment has been described elsewhere [6]. The resistance of the sample was measured as the temperature of the pressure bomb containing the sample was raised continuously. A carbon and platinum thermometer outside the pressure cell gave the temperature. To measure the thermal gradient between the thermometer and the sample, a run was made at 50 bar. No gradient was found for the lower anomaly and a temperature difference less than $5 \mathrm{~K}$ for the higher transition. Figures 1 and 2 show the variation of the resistivity with pressure for the two anomalies. In the inset we have plotted the variation of the critical temperature. $T_{\mathrm{c}}$

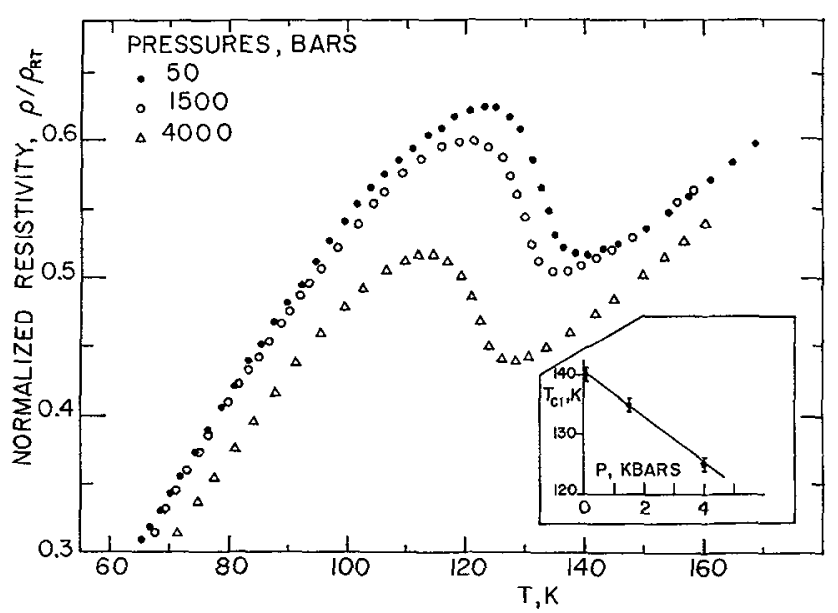

Frg. 1. - The normalized resistivity as a function of temperature for three different pressures for the higher temperature anomaly. Inset shows the pressure dependence of the critical temperature.

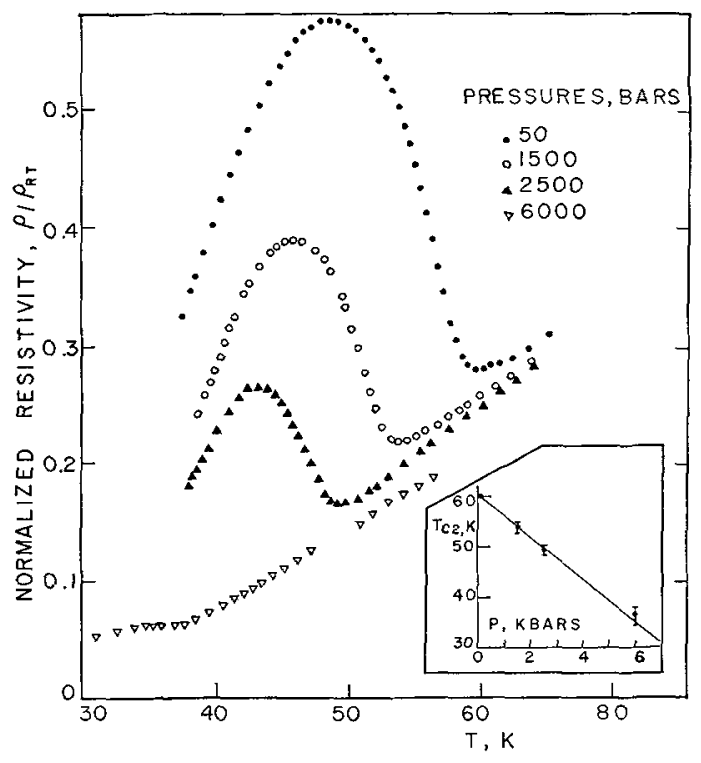

Frg. 2. - The normalized resistivity as a function of temperature for four different pressures for the lower temperature anomaly. Inset shows the pressure dependence of the critical temperature. varies linearly with pressure, and the slope is the same for the two transitions. We find $\mathrm{d} T_{\mathrm{c}} / \mathrm{d} p=4 \mathrm{~K} / \mathrm{kbar}$. The amplitude of the higher anomaly decreases with pressure and is reduced by $30 \%$ at $4 \mathrm{kbar}$. The reduction of the lower anomaly is much more important. The amplitude of the anomaly is more than $95 \%$ suppressed at $6 \mathrm{kbar}$. The formation of a CDW is determined by the competition between two terms in the free energy of the system : the strain energy, which increases with the formation of superlattice distortions, and the reduction in electronic energy resulting from the opening of gaps at the FS. The reduction in electronic energy increases with decreasing temperature. By applying pressure we expect a stiffening of the lattice, which increases the strain energy. Consequently, the critical temperature is lowered.

Electric field dependence of the conductivity was studied using dc and pulse currents. The details of the experiment will be published elsewhere [7]. The samples were typically $7 \times 0.05 \times 0.01 \mathrm{~mm}^{3}$ in size and were mounted on a quartz substrate inside a continuous helium gas-flow cryostat. For current densities below $10 \mathrm{~A} / \mathrm{mm}^{2}$ the dc technique was used. Selfheating of the sample became important above this value, and the pulsed technique extended the measurements to $100 \mathrm{~A} / \mathrm{mm}^{2}$. Pulses of $5 \mu$ s duration and $10 \mathrm{~ns}$ risetime were generated by a Chronetics PG-10, and the voltage signal across the sample displayed on a storage oscilloscope. Figures 3 and 4 show the suppression of the two peaks. Above $145 \mathrm{~K}$ ohmic behaviour is rigorously maintained. The phase transitions occur at the same temperatures $145 \mathrm{~K}$ and $59 \mathrm{~K}$ for all current den-

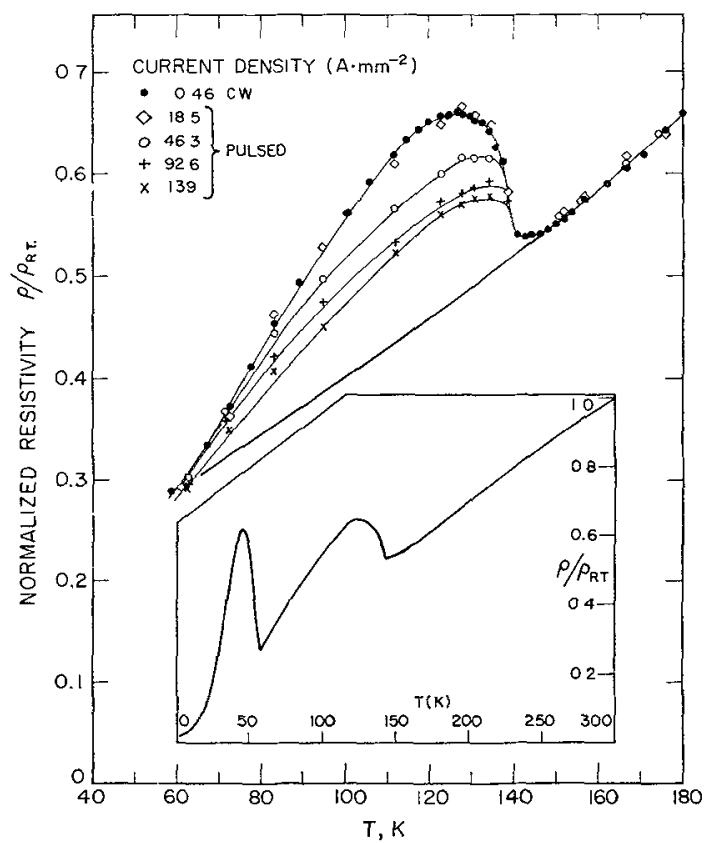

FIG, 3. - The normalized resistivity as a function of temperature at five current densities near the higher temperature anomaly. Inset shows the normalized resistivity of the lowest current density for the full temperature range. $\mathrm{CW}$ indicates non-pulsed experiments. 


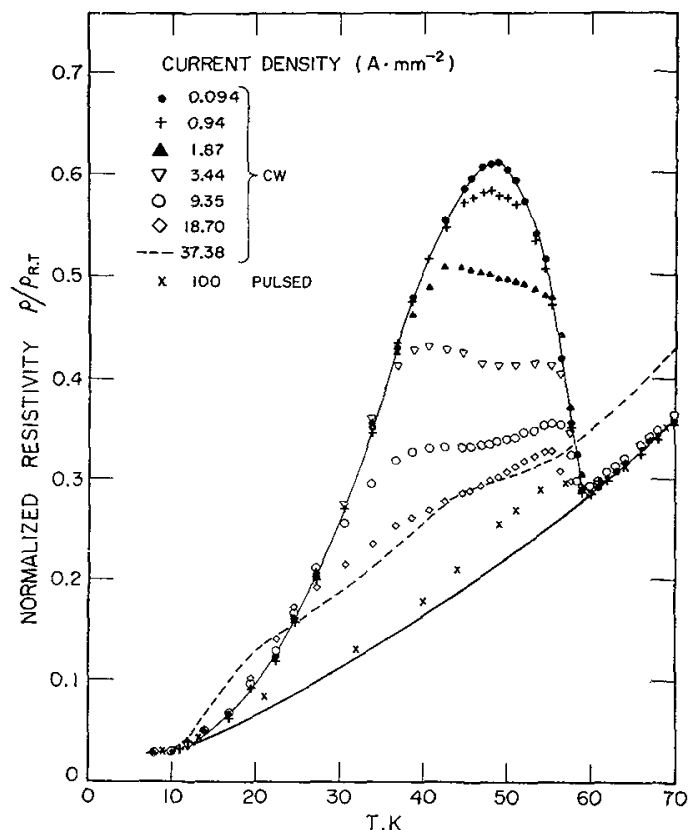

FiG. 4. - The normalized resistivity as a function of temperature near the lower temperature anomaly at seven current densities. The dashed line shows the effect of ohmic heating of the highest dc current density.

sities. The suppression of the lower peak is much more severe than that of the high temperature one. A $50 \%$ reduction requires only $3 \mathrm{~A} / \mathrm{mm}^{2}$ compared to $90 \mathrm{~A} / \mathrm{mm}^{2}$ for the higher peak. We want to emphasize the fundamental differences between these results and the pressure effect reported above. The suppression of the anomalies by the electric field does not affect the critical temperatures. The field affects the non-equilibrium properties of the sample as opposed to pressure, which alters the equilibrium state.

A high electric field induces Zener tunneling across the gap induced by the CDW. Using the electric breakdown equation [8] the conductivity may be written as

$$
\begin{gathered}
\sigma(T, E)=\sigma_{0}(T)+\sum_{n} \sigma_{n}(T) \mathrm{e}^{-E_{0 n}(T) / E} \\
E_{0 n}(T)=\pi \Delta_{n}^{2}(T) / \mid e j h v_{n}
\end{gathered}
$$

where the sum in eq. (1) is over the gaps, $\Delta_{n}$ is the $n$th half gap energy, and $v_{n}$ the component of the carrier velocity normal to the superlattice Bragg reflection plane to the FS in the absence of the $n$th gap. In the vicinity of the higher temperature anomaly at $123 \mathrm{~K}$ the data can be fitted to eq. (1) remarkably well to the highest applied field with just one term in the sum. Similarly, at $54 \mathrm{~K}$ the breakdown data can be well fitted with one term in eq. (1). At $49 \mathrm{~K}$ and $40 \mathrm{~K}$ two terms are required in the sum to give agreement with experiment. In figure 5 we have drawn the variation of $\sigma(T, E)-\sigma_{0}(T)$ as a function of $1 / E$ on a semi-log plot. The curves are eq. (1) with one or two terms in the summation.

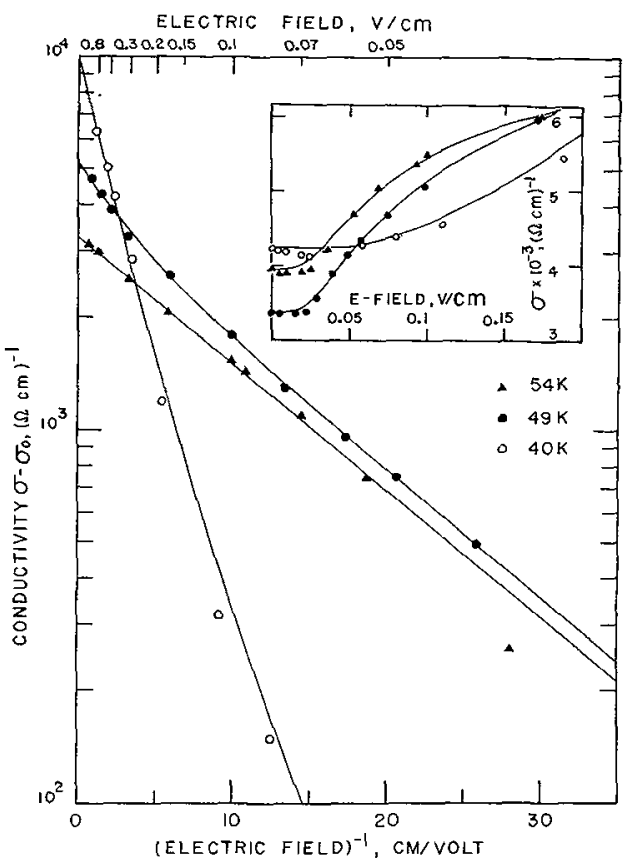

FIG. 5. - The fit of experimental data to Zener tunneling analysis. The solid lines are the best fit to the data using eq. (1) of three temperatures near the lower anomaly. For $54 \mathrm{~K}$ eq. (1) is fitted to the highest applied field with just one term in the sum. For $49 \mathrm{~K}$ and $40 \mathrm{~K}$ two terms are required. Inset shows the total conductivity versus electric field in the low field limit.

From this breakdown analysis it is possible to get a direct measurement of the gaps. Assuming a value of $v=10^{7} \mathrm{~cm} \mathrm{~s}^{-1}$ at all temperatures we obtain half gaps of $5.4 \times 10^{-5} \mathrm{eV}$ at $123 \mathrm{~K}$ and $1.3 \times 10^{-5} \mathrm{eV}$ at $54 \mathrm{~K}$. These gaps are extremely small and quite incompatible with a BCS type result

$$
\Delta=1.7 k T_{\mathrm{c}} \text {. }
$$

For $T=145 \mathrm{~K}$, eq. (3) gives $\Delta \sim 2 \times 10^{-2} \mathrm{eV}$ which is three orders of magnitude larger than the measured value. However, if both $\mathrm{s}$ and $\mathrm{d}$ electrons are present at the FS in $\mathrm{NbSe}_{3}$ (as in the case of $\mathrm{Cr}$ ) then the d-electron FS drives the superlattice phase transition while the $\mathrm{s}$ electrons dominate the transport properties. The weaker coupling of $s$ electrons to the soft phonon mode would result in a much smaller gap than given by eq. (3), and it may be possible to account in this way for the discrepancy in gap size.

Another possibility is that the transport properties are affected predominantly by second-order gaps, and it is these second order gaps that appear in eqs. (1) and (2). As pointed out by Falicov and Zuckermann [9] at the critical temperature a set of energy gaps appear at the Bragg diffraction planes at the $k$-values given by

$$
k=\frac{1}{2}(m G+n q) \quad m, n \text { integers }
$$

where $q$ is the spanning vector and $G$ the reciprocal lattice vector. The first order gap which follows eq. (3) is for $n=1$. But the second order gap $(n=2)$ in the 
reduced zone scheme may intercept and modify strongly the path of FS contributing to the transport properties. The magnitude of this second order gap is $\Delta^{2} / W$ [where $\Delta$ is given by eq. (3) and $W$ is the bandwidth of the carriers]. Assuming $W \sim 4 \mathrm{eV}$, then with $T_{\mathrm{c}}=145 \mathrm{~K}$ the second order half gap is of the order of $11 \times 10^{-5} \mathrm{eV}$ which roughly agrees with the measured value.

In conclusion, we have reported the pressure dependence of the critical temperatures at the onset of CDW in $\mathrm{NbSe}_{3}$. We have shown a very important nonlinear variation of the conductivity when a CDW is established. A Zener breakdown analysis gives directly the values of the gaps.

Acknowledgements. - We wish to acknowledge our indebtedness to A. Meerschaut, P. Molinie, and Prof. J. Rouxel, who provided the samples and to Prof. L. M. Falicov, who first suggested the possibility of Zener breakdown in $\mathrm{NbSe}_{3}$.

\section{References}

[1] Haen, P., Monceau, P., Tissier, B., Waysand, G., MeersChaut, A., Molinie, P. and Rouxel, J., Proceedings of the Low Temperature Conference LT 14 (Otaniomi, Finland), 1975, Vol. 5, p. 445.

[2] Chaussy, J., Haen, P., Lasuaunias, J. C., Monceau, P., Waysand, G., Boch, G., Waintal, A., Molinie, P. and Rouxer, J., Solid State Commun. in press.

[3] Wilson, J. A., DiSalvo, F. J. and Mahajan, S., Adv. Phys. 24 (1975) 117.

[4] Trego, A. L. and Mackintosh, A. R., Phys. Rev. 166 (1968) 495.
[5] Meerschaut, A. and RouxeL, J., J. Less Common Metals 39 (1975) 197.

[6] Waintal, A., Rouchy, J. and Draperi, A., Proceedings of the Vth International Conference on High Pressure and Technology, U. S. S. R., Moscow (1975).

[7] Monceau, P., Ong, N. P., Portes, A. M., Merrschaut, A. and Rouxel, J., Phys. Rev. Lett. 37 (1976) 602.

[8] Ziman, J. N., Principles of the Theory of Solids, second edition (Cambridge Univ. Press) 1972, Sec. 6-8.

[9] Falicov, L. M. and Zuckermann, M. J., Phys. Rev. 160 (1967) 372.

[10] Fruchart, R., private communication. 